\title{
Factores asociados a la sobrevida de los pacientes receptores de trasplante hepático por carcinoma hepatocelular según los criterios de Milán
}

\author{
Factors associated with the survival of liver transplant patients due to hepatocellular \\ carcinoma according to Milan criteria
}

Sergio Pacheco, Javier Rodríguez, Eduardo Briceño, Jorge Martínez, Juan F. Guerra y Nicolás Jarufe*

Departamento de Cirugía Digestiva, Pontificia Universidad Católica de Chile, Santiago, Chile

\begin{abstract}
Resumen
Objetivo: Determinar la sobrevida global de los pacientes sometidos a trasplante hepático (TH) por carcinoma hepatocelular $(\mathrm{CHC})$ siguiendo los criterios de Milán (CM), y analizar los factores asociados a la sobrevida. Método: Estudio de cohorte no concurrente. Se analizaron los pacientes sometidos a TH por CHC entre los años 2000 y 2016. Se realizó un análisis de los factores asociados a la sobrevida mediante Kaplan-Meier, test de log-rank y regresión de Cox. Se consideró significativo un valor de $p$ < 0.05. Resultados: Se realizaron $50 \mathrm{TH}$ por $\mathrm{CHC}$. El promedio de edad fue de $60.8 \pm 6.1$ años; 38 pacientes (76\%) fueron de sexo masculino. En el análisis multivariable, los factores asociados a la sobrevida fueron el cumplimiento de los CM (hazard ratio [HR]: 0.104; intervalo de confianza del 95\% [IC 95\%]: 0.017-0.637; $p=0.01$ ) y la ausencia de invasión vascular (HR: 0.050; IC 95\%: 0.008-0.306; $p$ < 0.01) en la biopsia del explante. Conclusión: La sobrevida de los pacientes sometidos a $\mathrm{TH}$ por $\mathrm{CHC}$ en nuestro centro es similar a lo reportado en la literatura internacional, y se encuentra determinada por el cumplimiento de los CM y la ausencia de invasión vascular en la biopsia del explante.
\end{abstract}

PALABRAS CLAVE: Carcinoma hepatocelular. Sobrevida. Trasplante hepático.

\begin{abstract}
Objective: To determine the overall survival of patients undergoing liver transplantation (LT) for hepatocellular carcinoma (HCC) following the Milan criteria (MC) and analyze factors associated with survival. Method: Non-concurrent cohort study. We analyzed patients undergoing LT for HCC between 2000 and 2016. An analysis of the factors associated with survival was carried out using Kaplan-Meier, log-rank test and Cox regression. $A$ value of $p<0.05$ was considered significant. Results: $A$ total of $50 L T$ were performed for HCC. The average age was $60.8 \pm 6.1$ years; 38 patients (76\%) were male. In the multivariate analysis, the factors associated with survival were compliance with CM (hazard ratio [HR]: 0.104; 95\% confidence interval [95\% Cl]: 0.017-0.637; $p=0.01$ ) and absence of vascular invasion (HR: 0.050; 95\% Cl: 0.008-0.306; $p<0.01$ ) in the explant biopsy. Conclusion: Survival of patients undergoing HT by HCC in our center is similar to that reported in the international literature, and is determined by the compliance of the CM and the absence of vascular invasion in the explant biopsy.
\end{abstract}

KEY WORDS: Hepatocellular carcinoma. Liver transplantation. Survival.

\footnotetext{
Correspondencia:

*Nicolás Jarufe

Diagonal Paraguay $362,4^{\circ}$

C.P. 8330077 , Santiago, Chile

E-mail: njarufe@med.puc.cl
}

Fecha de recepción: 15-07-2018

Fecha de aceptación: 20-09-2018

DOI: $10.24875 / C I R U .18000604$
Cir Cir. 2019;87:321-327

Contents available at PubMed www.cirugiaycirujanos.com 


\section{Introducción}

El carcinoma hepatocelular ( $\mathrm{CHC}$ ) es la sexta neoplasia maligna más frecuente en el mundo y la tercera más mortal ${ }^{1,2}$. Ocurre generalmente en pacientes con distintos grados de daño hepático crónico ${ }^{3}$. La cirrosis hepática producida por las hepatitis $\mathrm{B}$ y $\mathrm{C}$, o por el alcohol, es la enfermedad más asociada al $\mathrm{CHC}$, aunque en la última década la obesidad y la diabetes mellitus han emergido como importantes factores de riesgo $0^{4,5}$.

El trasplante hepático (TH) es la única opción terapéutica que permite tratar el tumor y la cirrosis hepática al mismo tiempo ${ }^{6,7}$. En este escenario, el TH ofrece buenos resultados en términos de sobrevida global y de sobrevida libre de enfermedad a largo plazo en pacientes seleccionados ${ }^{4,8,9}$. En el año 1996 fueron creados los criterios de Milán (CM) con el fin de seleccionar aquellos pacientes que pueden beneficiarse del TH con buena sobrevida y baja tasa de recidiva. Estos criterios son los siguientes: $\mathrm{CHC}$ único de 5 centímetros de diámetro o menos, o no más de tres tumores de 3 centímetros de diámetro o menos cada uno. Siguiendo estos criterios se ha obtenido una sobrevida a 4 años del $85 \%$ y una sobrevida libre de enfermedad del $92 \%{ }^{8}$. En los pacientes que reciben un $\mathrm{TH}$ y la biopsia del explante cumple los $\mathrm{CM}$, la sobrevida a 5 años es similar a la de aquellos que reciben un $\mathrm{TH}$ por causas no oncológicas ${ }^{1,4}$.

En el mundo en general, y en Chile en especial, la disponibilidad de donantes es escasa, por lo cual es indispensable tener criterios restrictivos con el fin de maximizar la utilización de órganos para trasplante. A partir del año 2002, en los EE.UU. se establece el puntaje Model for End Stage Liver Disease (MELD) como predictor para determinar la gravedad de los pacientes y su prioridad para el $\mathrm{TH}^{10}$, hecho que fue replicado en nuestro país en $2009^{11}$. En Chile, los pacientes con $\mathrm{CHC}$ que cumplen los $\mathrm{CM}$ ingresan a la lista de espera de TH con un puntaje MELD de 20 o el propio si este es mayor por la gravedad de la cirrosis.

En pacientes con $\mathrm{CHC}$ mayores de $2 \mathrm{~cm}$, la recomendación es realizar algún tratamiento previo al $\mathrm{TH}$ en caso de que este se demore más de 6 meses en lista de espera, con el fin de evitar la progresión y, por ende, la salida de los $\mathrm{CM}^{12}$. Dentro de estas terapias, las más utilizadas son la ablación tumoral, ya sea por radiofrecuencia o microondas, y la quimioembolización arterial. No existe clara evidencia de cuál de los procedimientos nombrados previamente es el más efectivo como terapia puente, pero su uso es bastante extendido ${ }^{13-15}$.

En nuestro país existe solo un reporte de los resultados del TH por CHC. Este estudio se realizó sobre una muestra de 29 pacientes, compuesta por enfermos del Hospital Clínico de la Pontificia Universidad Católica y la Clínica Alemana de Santiago ${ }^{4}$. Sin embargo, no existen reportes actualizados sobre esta patología, ni información de cómo ha variado la sobrevida de los pacientes sometidos a TH por CHC, dados los prolongados tiempos de espera para $\mathrm{TH}$ y el aumento en el número de TH realizados por esta causa en nuestro centro desde la aplicación del puntaje MELD para la priorización del TH.

El objetivo del presente estudio es determinar la sobrevida global de los pacientes sometidos a TH por CHC siguiendo los CM, y analizar los factores asociados a la sobrevida.

\section{Método}

Estudio de cohorte no concurrente. Se analizaron los pacientes sometidos a $\mathrm{TH}$ por $\mathrm{CHC}$ en el Hospital Clínico de la Pontificia Universidad Católica de Chile entre los años 2000 y 2016.

Los criterios de inclusión fueron: pacientes mayores de 15 años en que se realizó el TH por $\mathrm{CHC}$ que cumplieran los $\mathrm{CM}$ en los estudios imagenológicos preoperatorios y enfermos en los que se realizó el TH por razones distintas a $\mathrm{CHC}$, pero que en la biopsia del explante apareció incidentalmente un $\mathrm{CHC}$. Todos los TH fueron realizados mediante injertos obtenidos de donantes en muerte encefálica. Se excluyeron los pacientes que presentaban registros incompletos en las fichas clínicas o en los informes de anatomía patológica.

El diagnóstico de $\mathrm{CHC}$ se basó en la realización de dos pruebas imagenológicas (tomografía computada y resonancia magnética) y la determinación de las concentraciones plasmáticas de alfa-fetoproteína o la realización de biopsia. Mientras los pacientes se encontraban en lista de espera para $\mathrm{TH}$ se efectuaron exámenes imagenológicos cada 3 meses, para confirmar que siguieran cumpliendo los CM. Además, se realizaron terapias puente al TH según decisión individualizada del comité de $\mathrm{TH}$.

Se realizó una revisión de las fichas clínicas, electrónicas y de papel, de los informes de anatomía patológica y de los certificados en línea del Servicio de Registro Civil e Identificación. Se diseñó una base 
Tabla 1. Etiología del daño hepático crónico en los pacientes en estudio

\begin{tabular}{lcc}
\hline Etiología & $\mathbf{n}$ & $\%$ \\
\hline Esteatohepatitis no alcohólica & 17 & 34 \\
Consumo de alcohol & 17 & 34 \\
Virus de la hepatitis C & 8 & 16 \\
Cirrosis biliar primaria & 3 & 6 \\
Criptogénico & 2 & 4 \\
Hepatitis autoinmunitaria & 1 & 2 \\
Virus de la hepatitis B & 1 & 2 \\
Hemocromatosis & 1 & 2 \\
Total & 50 & 100 \\
\hline
\end{tabular}

Tabla 2. Terapias puente realizadas previamente al trasplante hepático

\begin{tabular}{lcc}
\hline Terapia & $\mathbf{n}$ & $\%$ \\
\hline Quimioembolización transarterial & 19 & 56.1 \\
Radiofrecuencia & 5 & 14.7 \\
Radiofrecuencia más quimioembolización transarterial & 4 & 11.7 \\
Radiofrecuencia más alcoholización & 3 & 8.8 \\
Alcoholización & 2 & 5.8 \\
Resección más quimioembolización transarterial & 1 & 2.9 \\
Total & 34 & 100 \\
\hline
\end{tabular}

de datos en el software Microsoft ${ }^{\circledR}$ Excel para el registro de los datos demográficos y clínicos, los informes de anatomía patológica, la mortalidad perioperatoria y la sobrevida de los pacientes en el seguimiento. El tiempo en lista de espera se consideró solo para pacientes sometidos a TH electivo. Se analizó la distribución de las variables continuas en estudio mediante el test de Shapiro-Wilk. Para la descripción de las variables continuas se utilizaron los estadígrafos media, desviación estándar, mediana y rango, según correspondiera. La descripción de las variables cualitativas se realizó mediante frecuencias absolutas y porcentajes.

Se realizó un análisis univariable de los factores asociados a la sobrevida mediante el método de Kaplan-Meier, comparando entre los grupos mediante el test de log-rank. Aquellos factores que en el análisis univariable presentaran un valor de $p<0.2$ fueron ingresados a un análisis multivariable mediante regresión de Cox para modelar los riesgos que afectaban la supervivencia de la población en estudio. Se consideró significativo un valor de $p<0.05$. Se utilizó el programa SPSS 22.0 para el análisis estadístico.

El presente estudio fue aprobado por el Comité de Ética de la Pontificia Universidad Católica de Chile. Ningún dato obtenido permitió la identificación de los pacientes en estudio.

\section{Resultados}

Durante el periodo en estudio se realizaron $50 \mathrm{TH}$ por $\mathrm{CHC}$. El promedio de edad fue de $60.8 \pm 6.1$ años, y 38 pacientes $(76 \%)$ fueron de sexo masculino. Las causas del daño hepático crónico más frecuentes fueron la esteatohepatitis no alcohólica y la ingesta de alcohol en 17 pacientes (34\%), cada una como se detalla en la tabla 1.

Cuarenta y siete pacientes (94\%) fueron sometidos a TH electivo; los restantes fueron sometidos a un TH de urgencia. De la totalidad de los pacientes, en 40 (80\%) la cirugía tuvo indicación por el diagnostico preoperatorio de $\mathrm{CHC}$, mientras que en $10(20 \%)$ se indicó por progresión de la enfermedad de base y el $\mathrm{CHC}$ se diagnosticó incidentalmente en la biopsia del explante. Todos los pacientes con $\mathrm{CHC}$ conocido cumplieron con los $\mathrm{CM}$ en el estudio imagenológico en el momento del TH. Cabe destacar que en 2 (4\%) enfermos se realizó una reducción de estadio del $\mathrm{CHC}$ mediante terapias puente hasta lograr completar los $\mathrm{CM}$.

De los pacientes en que se conocía el diagnóstico de $\mathrm{CHC}$ en el preoperatorio, en 34 (85\%) se realizó alguna terapia puente al TH. La terapia más frecuente fue la quimioembolización transarterial en 19 (56.1\%) casos, seguida por la radiofrecuencia en $5(14,7 \%)$. En algunos pacientes se utilizó más de una terapia, como se detalla en la tabla 2.

La mediana del puntaje MELD en el momento del TH fue de 12 (9-15), como puede verse en la tabla 3. Fallecieron 2 (4\%) pacientes en el posoperatorio, uno debido a un hemoperitoneo masivo y otro por una disfunción primaria del injerto. La mediana de la estadía hospitalaria fue de 13 días (3-71).

En la biopsia del explante, 44 (88\%) pacientes cumplieron los CM. Las características de los hallazgos histopatológicos se resumen en la tabla 4.

En el seguimiento, 2 (4\%) pacientes presentaron recidiva del $\mathrm{CHC}$, en un promedio de 18 meses tras el TH. Un paciente $(2 \%)$ presentó recidiva pulmonar y cerebral, mientras que el otro enfermo $(2 \%)$ presentó recidiva pulmonar y suprarrenal, siendo en ambos casos la causa de su posterior fallecimiento. 
Tabla 3. Características de los pacientes en estudio

\begin{tabular}{lc}
\hline Variables & $\mathbf{N}=\mathbf{5 0}$ \\
\hline Tiempo en lista de espera (meses) & $9(1-46)$ \\
MELD real en el momento del trasplante & $9(12-15)$ \\
hepático (puntaje) & \\
Índice de masa corporal del receptor $\left(\mathrm{kg} / \mathrm{m}^{2}\right)$ & $27.7(21.3-39)$ \\
Edad del donante (años) & $42.5 \pm 12.6$ \\
Tiempo operatorio (minutos) & $352(150-840)$ \\
Isquemia fría (minutos) & $506(187-840)$ \\
Isquemia caliente (minutos) & $31(17-79)$ \\
Glóbulos rojos transfundidos (unidades) & $3(2-5)$ \\
\hline
\end{tabular}

Tabla 4. Características histopatológicas

\begin{tabular}{lc}
\hline Variable & $\mathbf{N}=\mathbf{5 0}$ \\
\hline Número de tumores: & \\
- Uno & $35(70 \%)$ \\
- Dos & $10(20 \%)$ \\
- Tres & $2(4 \%)$ \\
- Cuatro & $2(4 \%)$ \\
- Seis & $1(2 \%)$ \\
Diámetro del tumor más grande $(\mathrm{cm})$ & $2.3(0.8-9)$ \\
Suma de diámetros máximos $(\mathrm{cm})$ & $2.9(0.8-9)$ \\
Nódulos satélites (sí) & $6(12 \%)$ \\
Invasión vascular & $2(4 \%)$ \\
\hline
\end{tabular}

La sobrevida de los pacientes al año y a los 5 años fue del 93.9 y el $76.5 \%$, respectivamente (Fig. 1). La tabla 5 muestra el análisis univariable de los factores asociados a la sobrevida. Las variables que resultaron con un valor de $p<0.2$ fueron el puntaje MELD en el momento del TH ( $p=0.11$ ), el cumplimiento de los CM en la biopsia del explante $(p<0.01)$ (Fig. 2$)$ y la ausencia de invasión vascular en la biopsia $(p<0.01)$ (Fig. 3). En la regresión de Cox (tabla 6), las únicas variables asociadas a una mayor sobrevida fueron el cumplimiento de los CM (hazard ratio [HR]: 0.104; intervalo de confianza del 95\% [IC 95\%]: 0.017$0.637 ; p=0.01)$ y la ausencia de invasión vascular (HR: 0.050; IC 95\%: 0.008-0.306; $p<0.01$ ) en la biopsia del explante.

\section{Discusión}

En el presente estudio, la sobrevida global de los pacientes sometidos a $\mathrm{TH}$ es prácticamente idéntica a lo reportado en la literatura nacional e internacional ${ }^{1,4}$. El cumplimiento de los CM y la ausencia de invasión vascular en la biopsia del explante se asociaron a una mayor sobrevida a largo plazo, como ha sido informado previamente $8,16,17$.

Las características demográficas y clínicas de los pacientes son similares a las señaladas en estudios previos $^{18}$. En la mayoría de los pacientes se conocía el diagnostico de $\mathrm{CHC}$ en el preoperatorio, y en casi la totalidad de ellos se realizó alguna terapia puente al TH. Esta wse recomienda en casos en que el TH se demorará probablemente más de 6 meses $^{12,13}$. En el presente estudio, la mediana de tiempo en lista de espera para TH fue de 9 meses, cifra superior a la presentada en otros estudios ${ }^{16}$ y a la recomendada en la literatura internacional, debido a la escasa cantidad de donantes en nuestro país. El cumplimiento de los CM se produjo en un $87.2 \%$ de los pacientes en estudio, lo que es mayor que lo obtenido en el estudio chileno previamente señalado ${ }^{4}$. Esto podría deberse a que el presente estudio se basa en una muestra homogénea de pacientes de un solo centro clínico, mientras que el estudio previo combinaba pacientes de dos centros distintos, con diferentes profesionales tratantes. Además, debe considerarse la probable influencia de la realización de exámenes imagenológicos seriados; en nuestro centro se realizaron cada 3 meses, lo que permitió excluir del TH a los pacientes que dejaran de cumplir los CM. Otro factor que cabe mencionar es la realización de terapias puente, que ayudaron a mantener a los pacientes cumpliendo los CM mientras se encontraban en lista de espera para TH.

En el presente estudio, un $20 \%$ de los pacientes presentó el CHC como hallazgo incidental en la biopsia. Esta situación no es infrecuente; la incidencia reportada varía entre un 4 y un $40 \%$ de los pacientes sometidos a TH por indicación no neoplásica ${ }^{19,20}$. La detección de $\mathrm{CHC}$ pequeños $(<3 \mathrm{~cm})$ es difícil debido a que el parénquima hepático del paciente cirrótico presenta fibrosis, nódulos regenerativos o displásicos, infiltración grasa y necrosis ${ }^{19}$. Los estudios previos señalan que la sobrevida de los pacientes con $\mathrm{CHC}$ incidental es similar a la de aquellos con $\mathrm{CHC}$ conocido en el preoperatorio ${ }^{21,22}$, lo cual fue confirmado por la presente investigación. Por lo tanto, es correcto reunir ambos grupos de pacientes para su estudio en conjunto.

En el presente estudio se realizó reducción del estadio tumoral para lograr cumplir con los CM en solo dos pacientes. La evidencia disponible señala que la sobrevida de los pacientes sometidos a $\mathrm{TH}$ en que se realizó reducción del estadio preoperatorio es similar a la de aquellos que se someten a TH cumpliendo los CM desde el inicio ${ }^{2,12,23}$. 
Tabla 5. Análisis univariable de los factores asociados a la sobrevida

\begin{tabular}{|c|c|c|c|c|}
\hline Variable & Categoría & Número (\%) & Sobrevida (meses) & $\mathbf{p}$ \\
\hline \multirow[t]{2}{*}{ Edad } & $<60$ años & $23(46 \%)$ & $129.9 \pm 15.2$ & 0.61 \\
\hline & $\geq 60$ años & $27(54 \%)$ & $96.3 \pm 10.7$ & \\
\hline \multirow[t]{2}{*}{ Sexo } & Masculino & $38(76 \%)$ & $123.8 \pm 15.2$ & 0.58 \\
\hline & Femenino & $12(24 \%)$ & $96.6 \pm 15.2$ & \\
\hline \multirow[t]{2}{*}{ Alfa-fetoproteína } & $<10 \mathrm{ng} / \mathrm{ml}$ & $43(86 \%)$ & $115.2 \pm 13.8$ & 0.48 \\
\hline & $\geq 10 \mathrm{ng} / \mathrm{ml}$ & $7(14 \%)$ & $103.7 \pm 15$ & \\
\hline \multirow[t]{2}{*}{$\mathrm{CHC}$ conocido } & No & $10(20 \%)$ & $110.8 \pm 11.7$ & 0.62 \\
\hline & Sí & $40(80 \%)$ & $118.2 \pm 13.6$ & \\
\hline \multirow[t]{2}{*}{ Tratamiento puente* } & Sí & $34(85 \%)$ & $114.6 \pm 17$ & 0.93 \\
\hline & No & $6(15 \%)$ & $104.8 \pm 23.8$ & \\
\hline \multirow[t]{2}{*}{ Lista de espera } & $<9$ meses & $25(50 \%)$ & $138.9 \pm 14.5$ & 0.35 \\
\hline & $\geq 9$ meses & $25(50 \%)$ & $95.2 \pm 10$ & \\
\hline \multirow[t]{2}{*}{ MELD al TH } & $<13$ & $25(50 \%)$ & $141.4 \pm 14.7$ & 0.11 \\
\hline & $\geq 13$ & $25(50 \%)$ & $98.2 \pm 13.2$ & \\
\hline \multirow[t]{2}{*}{ IMC receptor } & $<28 \mathrm{~kg} / \mathrm{m}^{2}$ & $25(50 \%)$ & $114.5 \pm 17.1$ & 0.32 \\
\hline & $\geq 28 \mathrm{~kg} / \mathrm{m}^{2}$ & $25(50 \%)$ & $118.9 \pm 12$ & \\
\hline \multirow[t]{2}{*}{ Edad del donante } & $<44$ años & $25(50 \%)$ & $119.2 \pm 15.5$ & 0.52 \\
\hline & $\geq 44$ años & $25(50 \%)$ & $106.6 \pm 9.9$ & \\
\hline \multirow[t]{2}{*}{ Tiempo operatorio } & $<360$ minutos & $31(62 \%)$ & $107.8 \pm 9.2$ & 0.95 \\
\hline & $\geq 360$ minutos & $19(38 \%)$ & $121.7 \pm 14$ & \\
\hline \multirow[t]{2}{*}{ Isquemia fría } & $<500$ minutos & $18(36 \%)$ & $120.5 \pm 12.5$ & 0.87 \\
\hline & $\geq 500$ minutos & $32(64 \%)$ & $77.4 \pm 6.8$ & \\
\hline \multirow[t]{2}{*}{ Isquemia caliente } & $<30$ minutos & $25(50 \%)$ & $120.1 \pm 14.6$ & 0.86 \\
\hline & $\geq 30$ minutos & $25(50 \%)$ & $98.7 \pm 10.6$ & \\
\hline \multirow[t]{2}{*}{ Unidades de GR } & $<3$ unidades & $23(46 \%)$ & $125.7 \pm 13.2$ & 0.55 \\
\hline & $\geq 3$ unidades & $27(54 \%)$ & $91.2 \pm 14.9$ & \\
\hline \multirow[t]{2}{*}{ CM en explante } & Sí & $44(88 \%)$ & $126.9 \pm 11.2$ & $<0.01$ \\
\hline & No & $6(12 \%)$ & $22.6 \pm 2.6$ & \\
\hline \multirow[t]{2}{*}{ Nódulos satélites } & Sí & $44(88 \%)$ & $25.8 \pm 0.7$ & 0.46 \\
\hline & No & $6(12 \%)$ & $123.7 \pm 11.3$ & \\
\hline \multirow[t]{2}{*}{ Suma de diámetros mayores } & $<2.9 \mathrm{~cm}$ & $25(50 \%)$ & $128.6 \pm 14.1$ & 0.36 \\
\hline & $\geq 2.9 \mathrm{~cm}$ & $25(50 \%)$ & $87.7 \pm 11.5$ & \\
\hline \multirow[t]{2}{*}{ Número de tumores } & 1 & $35(70 \%)$ & $112.6 \pm 12.9$ & 0.47 \\
\hline & $\geq 2$ & $15(30 \%)$ & $108.2 \pm 14.2$ & \\
\hline \multirow[t]{2}{*}{ Invasión vascular } & No & $48(96 \%)$ & $122.1 \pm 11.2$ & $<0.01$ \\
\hline & Sí & $2(4 \%)$ & $16.4 \pm 7.5$ & \\
\hline
\end{tabular}

*Solo considera pacientes con $\mathrm{CHC}$ conocido en el preoperatorio.

CHC: carcinoma hepatocelular; CM: criterios de Milán; GR: glóbulos rojos; IMC: índice de masa corporal; MELD: Model for End Stage Liver Disease; TH: trasplante hepático. 


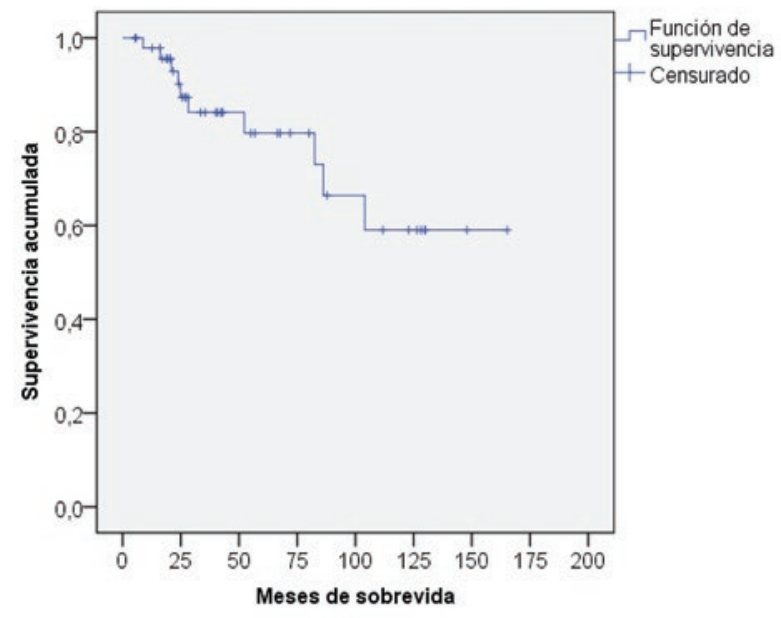

Figura 1. Curva de sobrevida de la totalidad de los pacientes en estudio.

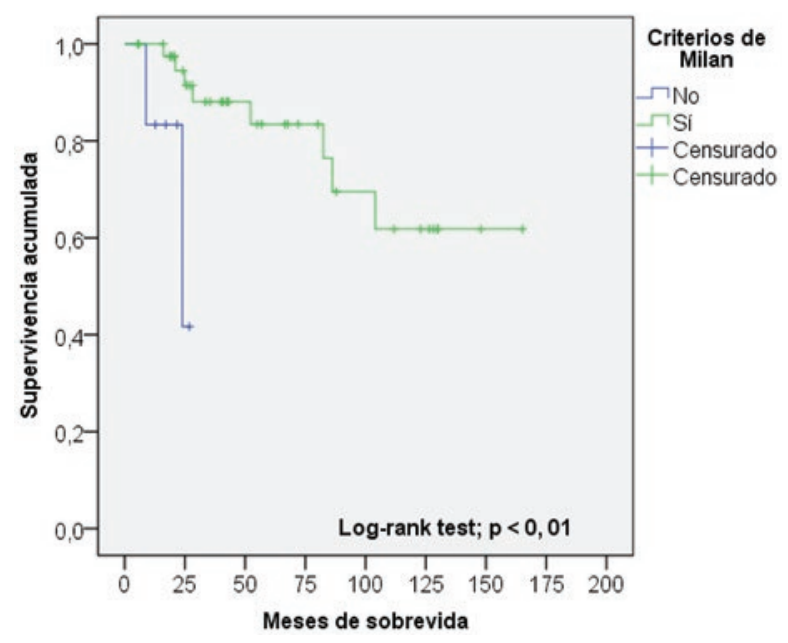

Figura 2. Comparación de la sobrevida entre pacientes que cumplieron o no los criterios de Milán en la biopsia del explante.

En el presente estudio no se encontró asociación entre la sobrevida a largo plazo y factores como los valores de alfa-fetoproteína y la transfusión de glóbulos rojos, lo cual difiere de investigaciones previas ${ }^{24,25}$. En cuanto a la transfusión de glóbulos rojos, podría deberse a la escasa cantidad de transfusiones realizadas en nuestros pacientes, pues la mediana fue de tres unidades.

Las limitaciones del presente estudio son su carácter observacional, retrospectivo y un tamaño muestral menor si se compara con series internacionales.

En conclusión, la sobrevida de los pacientes sometidos a $\mathrm{TH}$ por $\mathrm{CHC}$ en nuestro centro es similar a la reportada en la literatura internacional, y se encuentra determinada por el cumplimiento de los $\mathrm{CM}$ y la ausencia de invasión en la biopsia del explante.

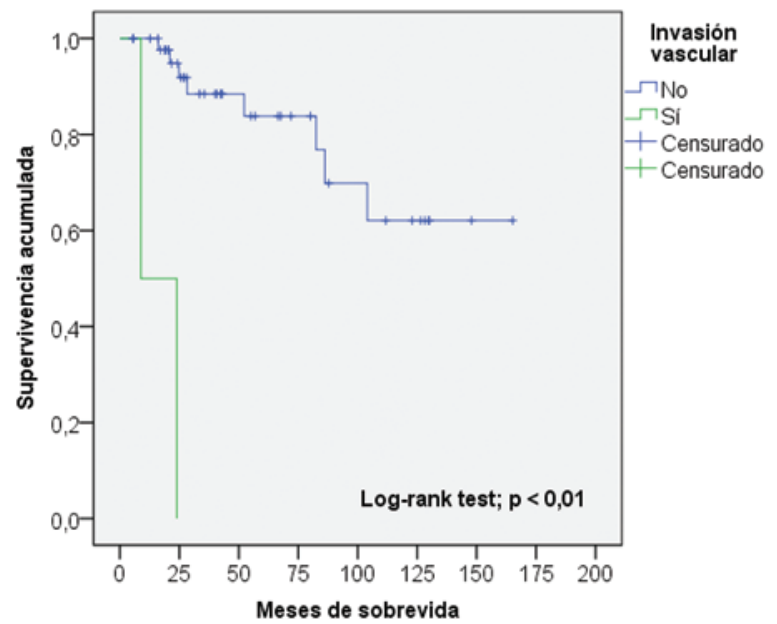

Figura 3. Comparación de la sobrevida entre pacientes que presentaron o no invasión vascular en la biopsia del explante.

Tabla 6. Análisis multivariable de los factores asociados a la sobrevida

\begin{tabular}{lcccc}
\hline Variable & Categoría & HR & IC 95\% & p \\
\hline MELD al TH & $<13$ & 0.269 & $0.056-1.291$ & 0.10 \\
& $\geq 13$ & & & \\
CM en explante & Sí & 0.104 & $0.017-0.637$ & 0.01 \\
& No & & & \\
Invasión vascular & No & 0.050 & $0.008-0.306$ & $<0.01$ \\
& Sí & & & \\
\hline
\end{tabular}

CM: criterios de Milán; HR: hazard ratio; IC 95\%: intervalo de confianza del 95\%; MELD: Model for End Stage Liver Disease; TH: trasplante hepático.

\section{Conflicto de intereses}

Los autores no refieren conflictos de intereses.

\section{Responsabilidades éticas}

Protección de personas y animales. Los autores declaran que para esta investigación no se han realizado experimentos en seres humanos ni en animales.

Confidencialidad de los datos. Los autores declaran que han seguido los protocolos de su centro de trabajo sobre la publicación de datos de pacientes.

Derecho a la privacidad y consentimiento informado. Los autores han obtenido el consentimiento informado de los pacientes y/o sujetos referidos en el artículo. Este documento obra en poder del autor de correspondencia. 


\section{Bibliografía}

1. Ravaioli M, Ercolani G, Neri F, Cescon M, Stacchini G, Del Gaudio M, et al. Liver transplantation for hepatic tumors: a systematic review. World J Gastroenterol. 2014;20:5345-52.

2. Agopian VG, Harlander-Locke M, Zarrinpar A, Kaldas FM, Farmer DG, Yersiz $\mathrm{H}$, et al. A novel prognostic nomogram accurately predicts hepatocellular carcinoma recurrence after liver transplantation: analysis of 865 consecutive liver transplant recipients. J Am Coll Surg. 2015;220:416-27.

3. Figueras J, Jaurrieta E, Valls C, Ramos E, Serrano T, Rafecas A, et al Resection or transplantation for hepatocellular carcinoma in cirrhotic patients: outcomes based on indicated treatment strategy. J Am Coll Surg. 2000;190:580-7.

4. Gabrielli M, Vivanco M, Hepp J, Martínez J, Pérez R, Guerra J, et al Liver transplantation results for hepatocellular carcinoma in Chile. Transplant Proc. 2010;42:299-301.

5. Wan $P$, Zhang J, Long X, Li Q, Xu N, Zhang M, et al. Serum levels of preoperative $\alpha$-fetoprotein and CA19-9 predict survival of hepatic carcinoma patients after liver transplantation. Eur J Gastroenterol Hepatol. 2014;26:553-61.

6. De Carlis L, Giacomoni A, Pirotta V, Lauterio A, Slim AO, Sammartino C, et al. Surgical treatment of hepatocellular cancer in the era of hepatic transplantation. J Am Coll Surg. 2003;196:887-97.

7. Tsuchiya K, Asahina Y, Tamaki N, Yasui Y, Hosokawa T, Ueda K, et al. Risk factors for exceeding the Milan criteria after successful radiofrequency ablation in patients with early-stage hepatocellular carcinoma. Liver Transplant. 2014;20:291-7.

8. Mazzaferro V, Regalia E, Doci R, Andreola S, Pulvirenti A, Bozzeti F, et al. Carcinomas in patients with cirrhosis. N Engl J Med. 1996;334:693-9.

9. Yao FY, Ferrell L, Bass NM, Watson JJ, Bacchetti P, Venook A, et al Liver transplantation for hepatocellular carcinoma: expansion of the tumor size limits does not adversely impact survival. Hepatology. 2001;33:1394-403.

10. Roma J, Balbi E, Pacheco-Moreira L, Zyngier I, Araujo A, Agoglia L, et al. Impact of Model for End-Stage Liver Disease Score on long-term surviva following liver transplantation for hepatocellular carcinoma. Transplant Proc. 2012;44:2423-7.

11. Sociedad Chilena de Trasplante. Guía Clínica de Trasplante Hepático. Editorial Sociedad Chilena de Trasplante. Santiago, Chile, 2009.

12. Clavien $P$, Lesurtel M, Bossuyt $P$. Recommendations for liver transplantation for hepatocellular carcinoma: an international consensus conference report. Lancet Oncol. 2012;13:1-21.
13. Graziadei IW, Sandmueller H, Waldenberger $P$, Koenigsrainer A, Nachbaur $\mathrm{K}$, Jaschke W, et al. Chemoembolization followed by liver transplantation for hepatocellular carcinoma impedes tumor progression while on the waiting list and leads to excellent outcome. Liver Transplant. 2003;9:557-63.

14. Sourianarayanane A, El-Gazzaz G, Sanabria JR, Menon KVN, Quintini $\mathrm{C}$, Hashimoto $\mathrm{K}$, et al. Loco-regional therapy in patients with Milan Criteria-compliant hepatocellular carcinoma and short waitlist time to transplant: an outcome analysis. HPB (Oxford). 2012;14:325-32.

15. Kim JM, Kwon CHD, Joh JW, Choi MS, Lee JH, Koh KC, et al. Effectiveness of locoregional therapy before living donor liver transplantation in patients with hepatocellular carcinoma who meet the milan criteria. Transplant Proc. 2012;44:403-8.

16. Nissen NN, Menon V, Bresee C, Tran TT, Annamalai A, Poordad F, et al. Recurrent hepatocellular carcinoma after liver transplant: identifying the high-risk patient. HPB (Oxford). 2011;13:626-32.

17. Hsieh C-H, Wei C-K, Yin W-Y, Chang C-M, Tsai S-J, Wang L-Y, et al. Vascular invasion affects survival in early hepatocellular carcinoma. Mol Clin Oncol. 2015;3:252-6.

18. Ramanathan R, Sharma A, Lee DD, Behnke M, Bornstein K, Stravitz RT, et al. Multimodality therapy and liver transplantation for hepatocellular carcinoma: a 14-year prospective analysis of outcomes. Transplantation. 2014;98:100-6.

19. Choi SH, Lee HH, Lee DS, Choi JH, Heo JS, Lee KW, et al. Clinicopathological features of incidental hepatocellular carcinoma in liver transplantation. Transplant Proc. 2004;36:2293-4.

20. Senkerikova R, Frankova S, Sperl J, Oliverius M, Kieslichova E, Filipova $\mathrm{H}$, et al. Incidental hepatocellular carcinoma: risk factors and long-term outcome after liver transplantation. Transplant Proc. 2014;46:1426-9.

21. Castillo E, Pelletier S, Kumer S, Abouljoud M, Divine G, Moonka D. Incidental hepatocellular carcinoma after liver transplantation: population characteristics and outcomes. Transplant Proc. 2009;41:219-21.

22. Shetty K, Timmins K, Brensinger C, Furth EE, Rattan S, Sun W, et al. Liver transplantation for hepatocellular carcinoma validation of present selection criteria in predicting outcome. Liver Transpl. 2011;10:911-8.

23. Sharr WW, Chan SC, Lo C-M. Section 3. Current status of downstaging of hepatocellular carcinoma before liver transplantation. Transplantation. 2014;97(Suppl 8):S10-7.

24. Ramos E, Dalmau A, Sabate A, Lama C, Llado L, Figueras J, et al. Intraoperative red blood cell transfusion in liver transplantation: influence on patient outcome, prediction of requirements, and measures to reduce them. Liver Transplant. 2003;9:1320-7.

25. Boin IFSF, Leonardi MI, Luzo ACM, Cardoso AR, Caruy CA, Leonardi LS. Intraoperative massive transfusion decreases survival after liver transplantation. Transplant Proc. 2008;40:789-91. 